Accepted for Publication in ApJ Letter, Preprint typeset using ATEXstyle

Preprint typeset using $\mathrm{LATEX}_{\mathrm{E}}$ style emulateapj v. 5/2/11

\title{
MAGNETOHYDRODYNAMIC TURBULENCE POWERED BY MAGNETOROTATIONAL INSTABILITY IN NASCENT PROTO-NEUTRON STARS
}

\author{
Youhei MASADA ${ }^{1}$, TOMOYA TAKIWAKI ${ }^{2}$ AND KeI KOTAKE ${ }^{3}$ \\ Accepted for Publication in ApJ Letter, Preprint typeset using LATEXstyle
}

\begin{abstract}
Magnetorotational instability (MRI) in a convectively-stable layer around the neutrinosphere is simulated by a three-dimensional model of supernova core. To resolve MRI-unstable modes, a thin layer approximation considering only the radial global stratification is adopted. Our intriguing finding is that the convectively-stable layer around the neutrinosphere becomes fully-turbulent due to the MRI and its nonlinear penetration into the strongly-stratified MRI-stable region. The intensity of the MRI-driven turbulence increases with magnetic flux threading the core, but is limited by a free energy stored in the differential rotation. The turbulent neutrinosphere is a natural consequence of rotating core-collapse and could exert a positive impact on the supernova mechanism.
\end{abstract}

Subject headings: Instabilities - turbulence - Supernovae: magnetic fields - stars: magnetic fields

\section{INTRODUCTION}

The magnetic field is not an exotic fuel for corecollapse supernovae (CCSNe) because it has self-excited and self-sustained natures and is an inevitable outcome of electrically-conducting fluid motions (e.g., Moffatt 1978). Once it is generated, it cannot be dissipated during CC$\mathrm{SNe}$ due to its long dissipation time $\left(\simeq 10^{15} \mathrm{sec}\right)$ (e.g. Masada et al. 2007). The mechanism of CCSNe should be thus studied self-consistently in the framework of magnetohydrodynamics (MHD) (e.g., Obergaulinger et al. 2006; Burrows et al. 2007; Takiwaki \& Kotake 2011; Winteler et al. 2012; Endeve et al. 2012; Mösta et al. 2014). See Kotake et al. (2012) and references therein for the MHD effect on the CCSNe.

The higher the magnetic field strength and the precollapse rotation rate, the more MHD effect becomes important in the supernova dynamics. At the postcollapse stage, the magnetic field is amplified by two processes: one is the field wrapping (i.e., $\Omega$-effect), and the other is so-called magnetorotational instability (MRI, see Balbus \& Hawley 1998). The later is highlighted here because it can amplify the magnetic field exponentially in the differentially rotating core, much faster than the linear amplification due to the $\Omega$-effect.

Akivama et al. (2003) were the first to point out that nascent protoneutron stars (PNSs) is generally subjected to the MRI. They predicted that, due to the MRI, the magnetic fields of $\sim 10^{15}-10^{16} \mathrm{G}$ can develop after the core-bounce, which is high enough to affect the supernova dynamics. However, the nonlinear properties of the MRI in the CCSNe has not been fully elucidated. The short wavelength of the MRI prevents from accurately capturing it in the global simulation.

To overcome this difficulty, local models are often used. Obergaulinger et al. (2009) reported local shearing-disk simulations to study the nonlinear development of the

\footnotetext{
${ }^{1}$ Department of Computational Science, Kobe University; Kobe 657-8501, Japan: E-mail: ymasada@harbor.kobe-u.ac.jp

${ }^{2}$ Division of Theoretical Astronomy, National Astronomical Observatory of Japan; Tokyo 181-8588

${ }^{3}$ Faculty of Science, Department of Applied Physics, Fukuoka University; Fukuoka 814-0180
}

MRI in the supernova core, and showed that the MRI amplifies the seed fields exceeding $10^{15} \mathrm{G}$ as estimated in Akivama et al. (2003). By local shearing box calculations of a small patch of the supernova core, Masada et al. (2012) presented a sub-grid scale model of the MRI-driven turbulence to predict the saturation amplitudes of the magnetic energy and turbulent stress. They also showed that the turbulent heating sustained by the MRI might play a crucial role in facilitating the neutrinoheating mechanism (see also Thompson et al. 2005).

Only by dropping the dimension, the MRI can be simulated even in the global model. A pioneering work by Sawai et al. (2013) showed for the first time that a submagnetar-class magnetic field was amplified by the MRI to the magnetar-class strength in the highest resolution axisymmetric global simulation to date. Furthermore, Sawai \& Yamada (2014) found by combining the neutrino transport approximately that the angular momentum transport by the MRI-amplified magnetic field contributes to the enhancement of the neutrino heating and eventually leads to neutrino-driven explosion.

The aim of this Letter is to comprehend, using a new semi-global model, three-dimensional (3D) evolution of the MRI in the collapsed-core with the global structure of MHD variables. To numerically resolve the MRI with the wavelength much shorter than the global scale, a thin layer approximation is adopted. The nonlinear evolution of the MRI in the stably stratified layer around the neutrinosphere and its dependence on the magnetic flux threading the core are studied quantitatively. The MRIsustained turbulent heating and the suitable condition for it are finally discussed.

\section{MODEL SETUP}

To resolve the MRI-unstable modes, we focus only on the equatorial region of the core and adopt a thin layer approximation with $r \gg h$, where $r$ and $h$ are the radius and height of the system. In Figure 1(a), our semi-global model is shown schematically. Under this approximation, the vertical gravity is neglected and thus the physical variables are homogeneous initially in the vertical direction. The computational domain covers $0 \leq r \leq 100[\mathrm{~km}]$ in the radius, $0 \leq \phi \leq 2 \pi$ in 

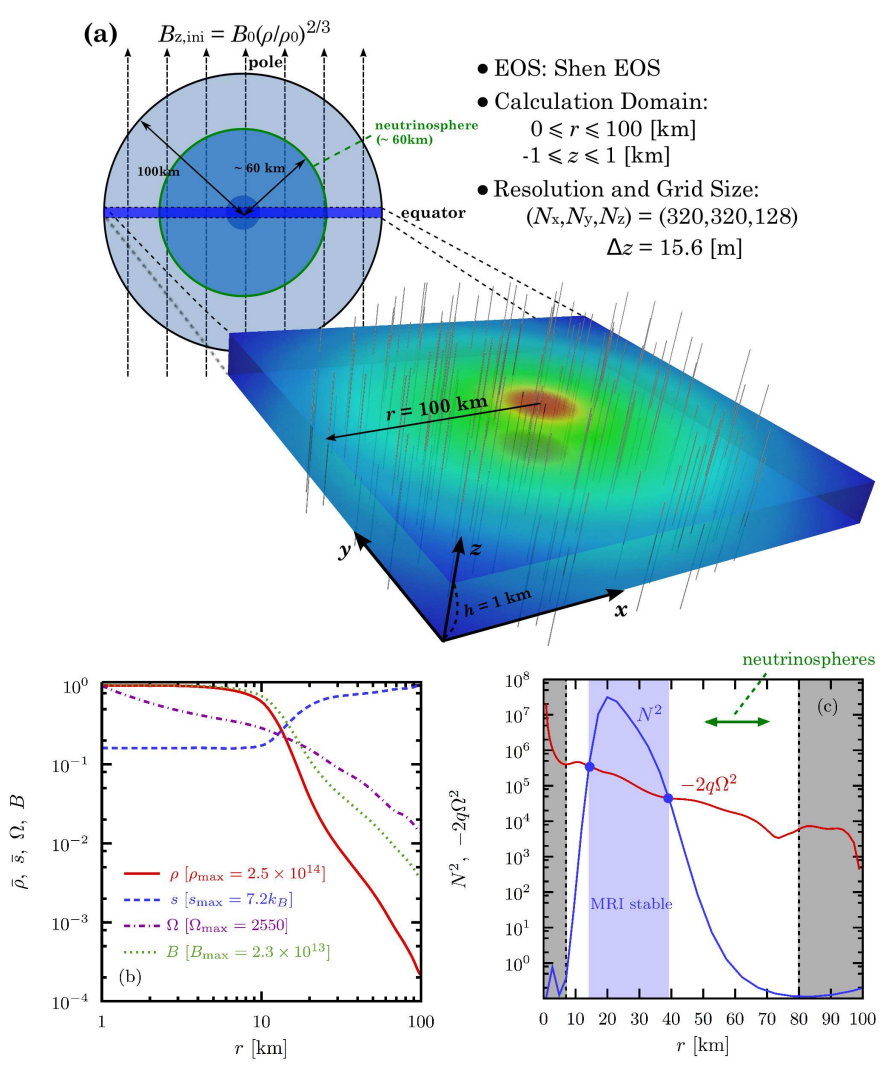

Fig. 1.- (a) Simulation setup. (b) Initial profiles of density (red solid), entropy (blue dashed), angular velocity (purple dash-dotted) and magnetic field (greed dotted) in our model. (c) Stability of the system to the MRI. The blue and red lines denote the LHS and RHS of equation (7). The positions of neutrinospheres are indicated by the horizontal arrow.

the azimuth, and $-1 \leq z \leq 1$ [km] in the height. The cylindrical structure of the thin-layer approximated supernova core is mapped onto the Cartesian domain with $L_{x}=L_{y}=200 \mathrm{~km}$ and $L_{z}=2 \mathrm{~km}$ to avoid the coordinate singularity.

The fundamental equations are compressible MHD equations written by

$$
\begin{aligned}
\frac{\partial \rho}{\partial t} & =-\nabla \cdot(\rho \boldsymbol{u}) \\
\frac{D \boldsymbol{u}}{D t} & =-\frac{\nabla P}{\rho}+\frac{\boldsymbol{J} \times \boldsymbol{B}}{c \rho}+\frac{\nabla \cdot\left(2 \rho \nu_{0} \boldsymbol{S}\right)}{\rho}+\boldsymbol{g}, \\
\frac{D \epsilon}{D t} & =-\frac{P \nabla \cdot \boldsymbol{u}}{\rho}+2 \nu_{0} \boldsymbol{S}^{2}+\frac{4 \pi \eta_{0} \boldsymbol{J}^{2}}{\rho c^{2}} \\
\frac{\partial \boldsymbol{B}}{\partial t} & =\nabla \times\left(\boldsymbol{u} \times \boldsymbol{B}-\frac{4 \pi \eta_{0} \boldsymbol{J}}{c}\right)
\end{aligned}
$$

with

$$
\boldsymbol{J}=\frac{c \nabla \times \boldsymbol{B}}{4 \pi}, S_{i j}=\frac{1}{2}\left(\frac{\partial u_{i}}{\partial x_{j}}+\frac{\partial u_{j}}{\partial x_{i}}-\frac{2}{3} \delta_{i j} \frac{\partial u_{i}}{\partial x_{i}}\right),
$$

where $\epsilon$ is the specific internal energy, $\boldsymbol{J}$ is the current density, and $S_{i j}$ is the strain rate tensor. The viscosity and magnetic diffusivity are represented by $\nu_{0}$ and $\eta_{0}$, respectively. We employ the equation of state based on the relativistic mean-field theory (Shen et al. 1998).

We set an initial equilibrium model based on a postbounce core from an axisymmetric hydrodynamic simulation of rotating core-collapse (see Takiwaki et al. 2014, for the method). About $100 \mathrm{~ms}$ after the core bounce, the shock wave has reached $\sim 200 \mathrm{~km}$, and the post-shocked region is settled into a quasi-hydrostatic structure. The radial distribution of the hydrodynamic variable along the equator of the simulation is extracted, and the stratified supernova core is reconstructed within our model. By assuming the gravity force balancing with the pressure gradient and centrifugal forces, a cylindrically symmetric hydrostatic structure is retained.

The radial profiles of the density, entropy, and angular velocity adopted as the initial setting are shown in Figure 1(b) by red solid, blue dashed, and purple dashdotted lines. They are normalized by their maximum values $\left(\rho_{\max }=2.5 \times 10^{14} \mathrm{~g} / \mathrm{cm}^{3}, s_{\max }=7.2 k_{B}\right.$ and $\left.\Omega_{\max }=2500 \mathrm{rad} / \mathrm{sec}\right)$. The angular velocity adopted here corresponds to that of a rapidly rotating PNS (e.g., Ott et al. 2006). The initial configuration of the magnetic field is given by

$$
B_{z}(r)=B_{0}\left[\rho(r) / \rho_{0}\right]^{2 / 3},
$$

where $\rho_{0}$ is the density at the reference point $r=30 \mathrm{~km}$ and $B_{0}$ is the magnetic field there. The dependence of the MRI-driven turbulence on $B_{0}$ is studied in $\S 3.2$.

In our simulation, the profile of the electron fraction $Y_{e}$ is assumed to be constant with time from the initial value because neutrino transport is not solved. The convective stability is thus determined only by the entropy gradient. Since the effect of the $Y_{e}$-gradient on the MRI is much weaker than that of the entropy gradient in the region of our interest $(8 \mathrm{~km} \lesssim r \lesssim 80 \mathrm{~km})$, we anticipate that, at least, the conclusion of this work is independent from it.

The stability condition of the stratified system to the MRI is written by (e.g., Balbus \& Hawley 1994; Masada et al. 2006)

$$
N^{2}>-2 q \Omega^{2}
$$

with

$$
N^{2} \equiv-\frac{1}{\gamma \rho} \nabla P \cdot \nabla \ln \left(P \rho^{-\gamma}\right), \quad q=\frac{\partial \ln \Omega}{\partial \ln r},
$$

where $\Omega$ is the angular velocity and $q$ is the shear rate. The LHS is the squared Brunt-Väisäla frequency due to the entropy gradient and the RHS is the destabilization effect by the MRI. In Figure 1(c), the LHS and RHS of equation (7) evaluated from the initial profiles are shown as a function of the radius. Our model comprises three characteristic regions: inner and outer MRI-unstable regions $(r \lesssim 15 \mathrm{~km}$ and $40 \mathrm{~km} \lesssim r$ ), and middle MRIstable region $(15 \mathrm{~km} \lesssim r \lesssim 40 \mathrm{~km}$ ). The horizontal arrow indicates the positions of (energy-integrated) neutrinospheres, which is smallest for heavy neutrinos $(\sim 50$ $\mathrm{km})$ and largest $(\sim 70 \mathrm{~km})$ for electron neutrinos. The neutrinospheres are located in the MRI-unstable region initially.

All the variables are assumed to be periodic in the vertical direction, whereas stress-free and perfect conductor boundary conditions are used in the horizontal direction for the velocity and magnetic fields. To reduce the artifacts due to the Cartesian geometry, the profile of the angular velocity is fixed in buffer regions denoted by gray shade in Figure 1(c) $(r<8 \mathrm{~km}$ and $r>80 \mathrm{~km})$. Furthermore, we assume 100 times larger magnetic diffusivity in the buffer region than that in the region of 

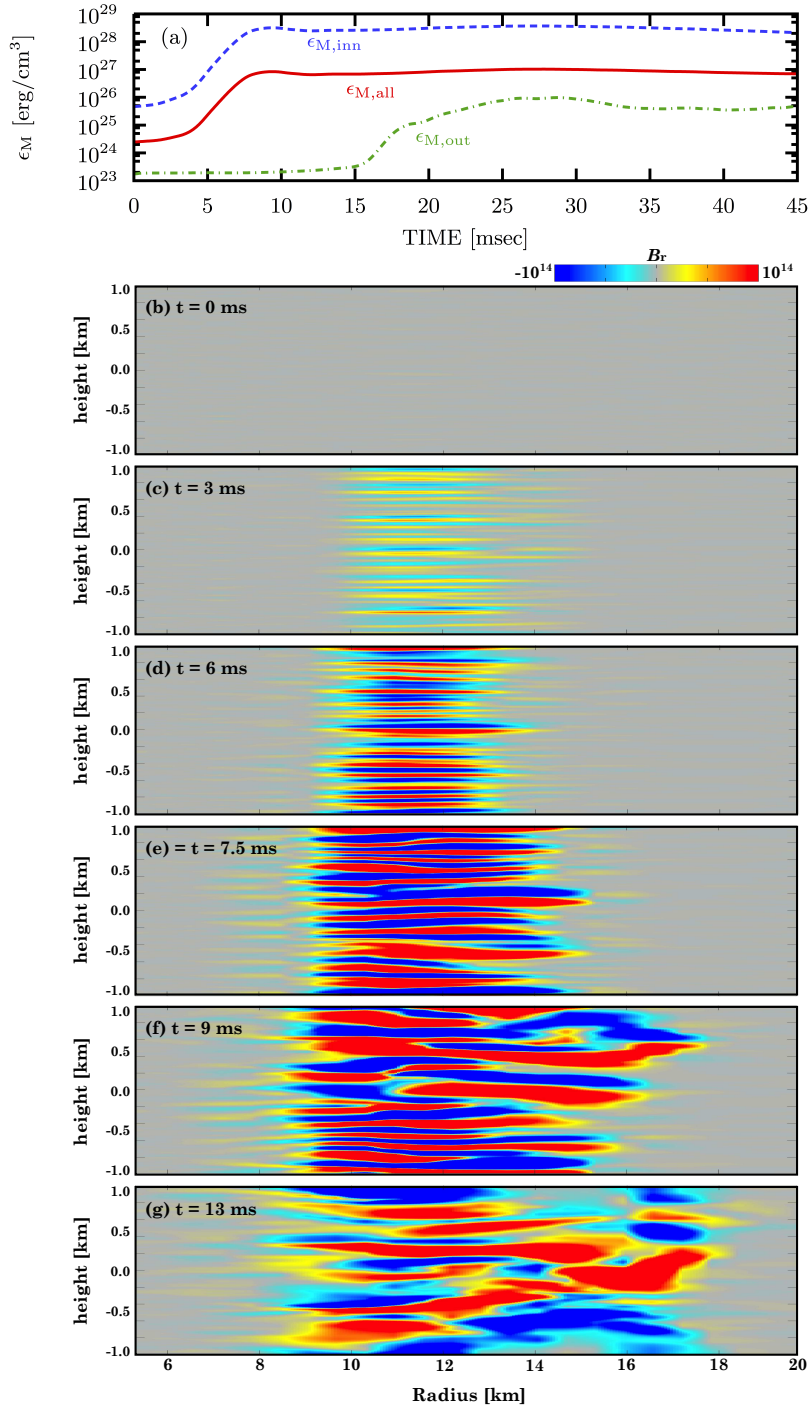

FIG. 2.- (a) Time series of volume-averaged magnetic energy [entire volume (red solid), inner volume (blue dashed), and outer volume (green dash-dotted) averages]. (b)-(f) Snapshots of $B_{r}$ in the inner MRI-unstable region on the $r-z$ cutting plane.

$8 \mathrm{~km} \leq r \leq 80 \mathrm{~km}$. The MRI-driven turbulence is thus artificially quenched in the buffer region.

The fundamental equations are solved by the secondorder Godunov-type finite-difference scheme which employs an approximate MHD Riemann solver (Sano et al. 1998; Masada et al. 2012). The magnetic field is evolved with CMoC-CT method (Evans \& Hawlev 1988; Clarke 1996). The viscosity and resistivity are chosen as $\nu_{0}=$ $\eta_{0}=10^{12} \mathrm{~cm}^{2} / \mathrm{sec}$ in the buffer region, and set to zero in the other region. The spatial resolution of $\left(N_{x}, N_{y}, N_{z}\right)$ $=(320,320,128)$ is adopted for all the simulation runs. The grid convergence of our model will be studied carefully in a subsequent paper. A small (1\%) random perturbation relative to the unperturbed initial velocity field is added when the calculation starts.

\section{SIMULATION RESULTS}

\subsection{Fiducial Run with $B_{0}=10^{12} \mathrm{G}$}

The temporal-evolution of the MRI in a fiducial model with $B_{0}=10^{12} \mathrm{G}$ is elaborated. Shown in Figure 2 (a) is the time series of volume-averaged magnetic energy. The red solid, blue dashed and green dash-dotted lines denote the entire volume, inner volume $(8 \mathrm{~km} \leq r \leq 15 \mathrm{~km})$, and outer volume $(15 \mathrm{~km} \leq r \leq 80 \mathrm{~km})$ averages, respectively. After the initial exponential growth phase, the magnetic energy is saturated at $t \simeq 10 \mathrm{~ms}$. By comparing the inner and outer magnetic energies, it is found that the early evolution is dominated by the MRI growth in the inner region. The delay of the MRI growth in the outer MRI-unstable region would be because our simulation cannot sufficiently capture the most unstable MRI wavelength there.

Panels (b)-(f) in Figure 2 present the snapshots of the radial magnetic field $\left(B_{r}\right)$ in the inner MRI-unstable region on the $r-z$ cutting plane at $t=0-12.5 \mathrm{~ms}$, with the red (blue) tone denoting the positive (negative) $B_{r}$. As seen in earlier studies, the evolution and disruption of the channel structure are observed (e.g., Obergaulinger et al. 2009; Masada et al. 2012). Since the convective motion does not develop in the stably stratified region, the secondary instabilities, such as parasitic instability, are responsible for the disruption of the channels (see, e.g., Obergaulinger et al. 2009; Sawai et al. 2013, for the effects of the secondary instabilities and background flow motion on the MRI).

The growth of the MRI in the outer region becomes prominent after $t \simeq 15 \mathrm{~ms}$ [green dash-dotted line in Figure 2(a)]. Since the magnetic energy stored in the outer region is small compared to the inner region, it gives a minor contribution to the total magnetic energy. However, the material mixing by the MRI-driven turbulence plays a crucial role in reducing the entropy gradient and thus in developing the fully-turbulent PNS surface.

Figure 3 is the $3 \mathrm{D}$ visualization of the magnetic field line in the late evolutionary phase after $t \simeq 10 \mathrm{~ms}$ (view from the $z$-direction). Panels (a)-(d) correspond to the sequential snapshots. The magnetic field line with the color denoting its absolute strength is superimposed on the volume visualization of the density. The red and white dashed lines in the panel (a) are the circles with $r=15$ and $40 \mathrm{~km}$ as a reference.

In the late evolutionary stage, the MRI begins to grow in the outer MRI-unstable region $(r \gtrsim 40 \mathrm{~km})$ at around $t \simeq 15 \mathrm{~ms}$ [panel (a)]. Intriguingly, as time advances, the MRI-turbulent region spreads radially-inward [panels (b) \& (c)], and penetrates into the middle MRI-stable region [panel (d)]. The turbulent mixing which can flatten the entropy gradient would be responsible for it. As a result, whole the convectively stable region around the neutrinosphere is overwhelmed with the MHD turbulence.

The multistage evolution of the MRI can be seen also in the energy spectra. In Figure 4, the Fourier spectra of the magnetic energy, averaged over the azimuth and a given time span at (a) $r=10 \mathrm{~km}$ (inner MRI-unstable region), (b) $r=30 \mathrm{~km}$ (middle MRI-stable region), and (c) $r=60 \mathrm{~km}$ (outer MRI-unstable region), are shown. The horizontal axis represents the vertical wavenumber $k$ normalized by $k_{c} \equiv 2 \pi / L_{z}$. The different lines correspond to different time spans. The black dashed line is the reference slope of $\propto k^{-5 / 3}$.

The magnetic energy inversely cascades from the small scale to the larger scale as time passes. In the inner MRI-unstable region [panel (a)], it begins to evolve immediately after the calculation starts and a saturated 

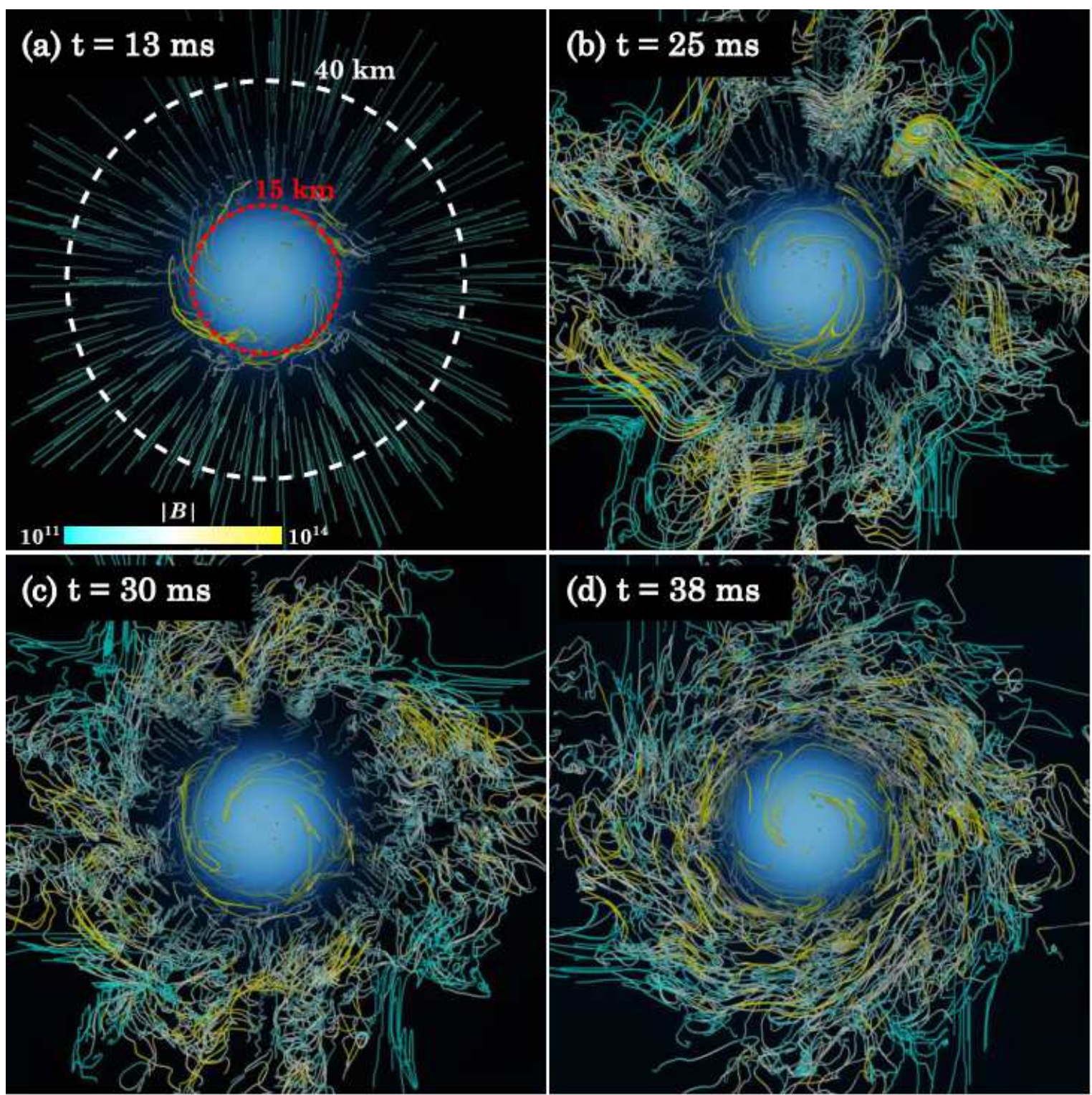

FIG. 3.- 3D visualization of the magnetic field line. Panels (a)-(d) correspond to the sequential snapshots when $t=13-38$ ms. The magnetic field line with the color denoting its absolute strength is superimposed on the volume visualization of the density.

MRI-turbulent state is achieved at $t \simeq 10 \mathrm{~ms}$. After $t \simeq 10 \mathrm{~ms}$, the outer MRI-unstable region begins to be destabilized [panel (c)]. The middle MRI-stable region is subsequently destabilized after $t \simeq 20 \mathrm{~ms}$ and is finally settled into the turbulent state at $t \simeq 40 \mathrm{~ms}$ [panel (b)].

\subsection{Dependence on $B_{0}$}

The property of the saturated MRI-driven turbulence is examined by varying $B_{0}$ from $10^{11}$ to $5 \times 10^{13} \mathrm{G}$ without changing the initial equilibrium model.

Figure 5 (a) shows the $B_{0}$-dependence of the mean magnetic energy density $\left\langle\left\langle\epsilon_{M}\right\rangle\right\rangle$, where the single angular brackets denote the volume average spanning in the range of $r_{\text {in }} \leq r \leq r_{\text {out }}$ and the additional angular brackets denote the time average in the range of $t_{s} \leq t \leq t_{e}\left[r_{\text {in }}\right.$ $\left(r_{\text {out }}\right)$ is $10 \mathrm{~km}(80 \mathrm{~km})$ and $t_{s}\left(t_{e}\right)$ is $30 \mathrm{~ms}(40 \mathrm{~ms})$ for all the models]. The red circle represents the MRI-turbulent model and the green cross is the model in which the MRI does not grow. The orange dotted line is the reference slope with $\propto B_{0}^{2}$. The expected magnetic energy stored in the full spherical shell of $r_{\text {in }} \leq r \leq r_{\text {out }}$, which is defined by

$$
E_{\text {mag }}=\frac{4}{3} \pi\left(r_{\text {out }}^{3}-r_{\text {in }}^{3}\right)\left\langle\left\langle\epsilon_{M}\right\rangle\right\rangle,
$$

is presented in the right axis just for the reference.

In the range $B_{0} \ll 10^{13} \mathrm{G},\left\langle\left\langle\epsilon_{M}\right\rangle\right\rangle$ is proportional to $B_{0}^{2}$. This is similar to the recent studies of the MRI in the stratified accretion disk (e.g., Suzuki et al. 2010). The dependency becomes weaker with the increase of $B_{0}$ and $\left\langle\left\langle\epsilon_{M}\right\rangle\right\rangle$ hits the ceiling at $\epsilon_{\text {upper }} \simeq 4 \times 10^{27} \mathrm{erg} / \mathrm{cm}^{3}$ in the range $B_{0} \gtrsim 10^{13} \mathrm{G}$. Except for the most strongly magnetized model in which the MRI does not grow, whole the convectively stable region around the neutrinosphere is overwhelmed with the MHD turbulence.

The upper threshold $\epsilon_{\text {upper }}$ seems to be determined by the free energy stored in the differential rotation. By tak- 

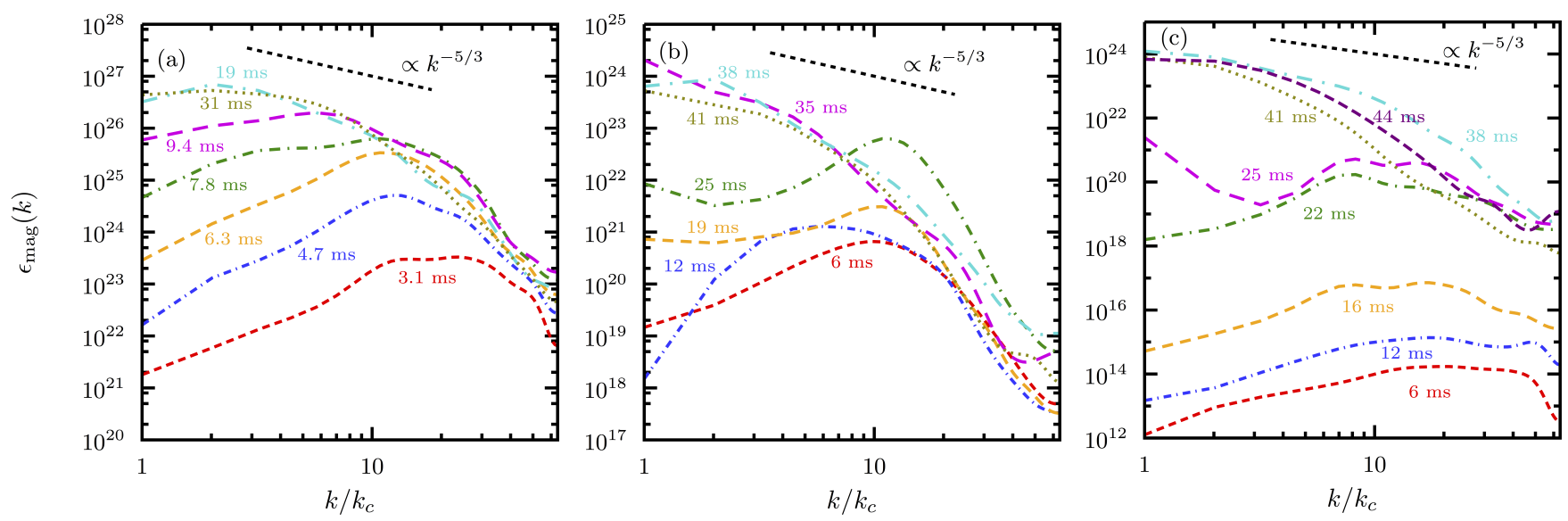

FIG. 4.- Fourier spectra of the magnetic energy at (a) $r=10 \mathrm{~km}$, (b) $r=30 \mathrm{~km}$, and (c) $r=60 \mathrm{~km}$, which are averaged over the azimuth and a given time span. The horizontal axis is the vertical wavenumber $k$ normalized by $k_{c} \equiv 2 \pi / L_{z}$. The black dashed line is the reference slope of $\propto k^{-5 / 3}$.

ing the initial rotation profile, the volume-averaged shear energy $\left\langle\epsilon_{\text {shear }}\right\rangle$, which can be tapped for the amplification of the magnetic field, is evaluated as

$$
\left\langle\epsilon_{\text {shear }}\right\rangle \equiv\left\langle\frac{1}{2}\left(\frac{\Delta \Omega}{\Omega}\right)^{2} \rho v_{\phi}^{2}\right\rangle \simeq 4 \times 10^{27} \mathrm{erg} / \mathrm{cm}^{3},
$$

where $\Delta \Omega \equiv(\partial \Omega / \partial r) \mathrm{d} r$ is the rotational shear (see, e.g., Spruit 2008). $\left\langle\epsilon_{\text {shear }}\right\rangle$ is presented by the blue dashed line in the panel (a). There is a good agreement between $\epsilon_{\text {upper }}$ and $\left\langle\epsilon_{\text {shear }}\right\rangle$. This suggests that the convectivelystable layer around the neutrinosphere becomes fullyturbulent when the condition

$$
\left\langle\epsilon_{M, \text { init }}\right\rangle \lesssim\left\langle\epsilon_{\text {shear }}\right\rangle
$$

is fulfilled, where $\epsilon_{M \text {,init }}$ is the initial magnetic energy density. The most strongly magnetized model does not meet this precondition. This would be the reason why the MRI-turbulence does not grow in that model.

The upper threshold for the saturated magnetic energy is a main difference between the MRI in CCSNe and that in the accretion disk. In the supernova core, the force balance is mainly achieved between the gravity and the pressure gradient force unlike the accretion disk in which the gravity is balanced with the centrifugal force. Since the free energy stored in the differential rotation is much lower than the thermal energy in the supernova core, the saturated magnetic energy is constrained predominantly by the shear energy rather than by the thermal energy.

The MRI-driven turbulence sustains the turbulent heating around the neutrinosphere. Figure 5 (b) shows the $B_{0}$-dependence of the MRI-luminosity $\mathcal{L}_{\mathrm{MRI}}$, which is equivalent to the turbulent heating rate and is defined by

$$
\mathcal{L}_{\mathrm{MRI}}=\int_{r_{\mathrm{in}}}^{r_{\mathrm{out}}} \int_{t_{s}}^{t_{e}} 4 \pi r^{2} w_{\mathrm{M}} q \Omega \mathrm{d} r \mathrm{~d} t,
$$

where $w_{\mathrm{M}} \equiv-B_{r} B_{\phi} / 4 \pi$ is the turbulent Maxwell stress (see Thompson et al. 2005; Masada et al. 2012). Note that the spherical symmetry is assumed here. The red circle denotes the model dominated by the MRI-driven turbulence, and the green cross is the model in which the MRI does not grow.

$\mathcal{L}_{\mathrm{MRI}}$ is increased with $B_{0}$ in the regime $B_{0} \lesssim 10^{13} \mathrm{G}$. However, in the most strongly magnetized model, the turbulent heating is drastically suppressed because the MRI-driven turbulence does not develop. This suggests that, in the rapidly rotating PNS assumed in this work, the pulsar-sized relatively weak poloidal magnetic field $\left(10^{12}-10^{13} \mathrm{G}\right)$ is the most suitable for the MRI-sustained turbulent heating and then provides $\mathcal{L}_{\mathrm{MRI}} \simeq 10^{51} \mathrm{erg} / \mathrm{sec}$ in the supernova core.

By extrapolating the simulation result of the rapidly rotating PNS, we finally evaluate $\mathcal{L}_{\mathrm{MRI}}$ expected in PNSs with extremely rapid rotation. When assuming $w_{\mathrm{M}} \propto$ $\Omega^{2}, \mathcal{L}_{\mathrm{MRI}}$ should be proportional to $\Omega^{3}$ from the equation (12). In addition, equation (11) suggests that the maximum value of $B_{0}$ for enabling the MRI-driven turbulence is proportional to $\Omega$. According to Burrows et al. (2007) and Takiwaki et al. (2009), the most rapidly rotating PNS is expected to have 3 times higher $\Omega_{0}$ than that of our simulation model $\left(\Omega_{0}=2500 \mathrm{rad} / \mathrm{s}\right)$. To infer an upper bound of $L_{\mathrm{MRI}}$, we plot the blue-dashed line in Figure 5 (b), which corresponds to the extrapolated model. The MRI-luminosity is sensitive to $\Omega$ and would have larger impact on the supernova dynamics in the faster spinning PNSs.

\section{SUMMARY}

In this Letter, we studied the 3D evolution of the MRI by a semi-global model simulating the supernova core. The nonlinear property of the MRI-driven turbulence in the stably stratified layer around the neutrinosphere and its dependence on the initial magnetic flux were quantitatively elucidated.

It was found that the MRI-driven turbulence excited in inner and outer MRI-unstable regions gradually erodes the strongly-stratified middle MRI-stable region. Finally, whole the convectively stable region around the neutrinosphere was overwhelmed with the MHD turbulence. The intensity of the saturated MRI-driven turbulence in the PNS was affected by the magnetic flux threading the collapsed core: while the magnetic energy sustained by the MRI-driven turbulence was proportional to $B_{0}^{2}$ in the range $B_{0} \ll 10^{13} \mathrm{G}$, it is limited by the free energy stored in the differential rotation when $B_{0} \gtrsim 10^{13} \mathrm{G}$.

Our results indicate that the turbulent neutrinosphere is a natural consequence of rotating core-collapse even in the absence of convection. In addition, the MRIsustained turbulent heating is the most effective when 

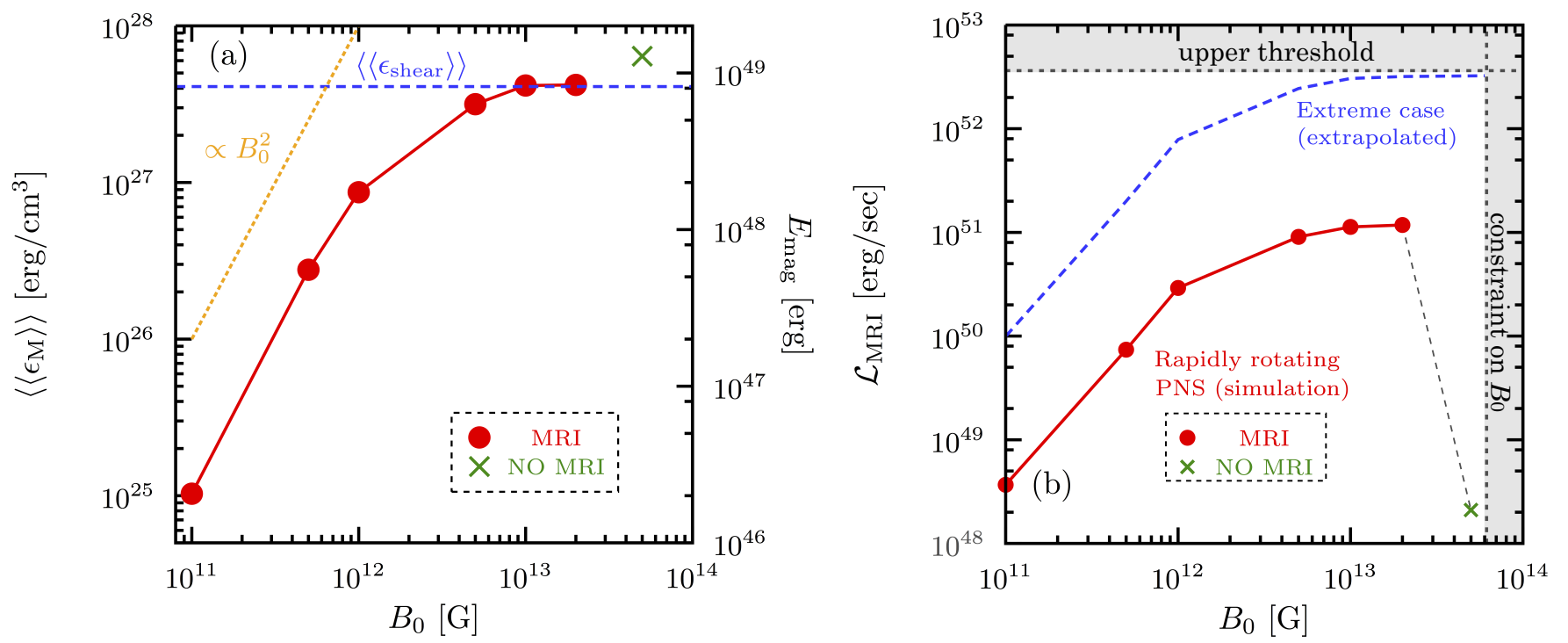

FIG. 5.- (a) $\left\langle\left\langle\epsilon_{M}\right\rangle\right\rangle$ as a function of $B_{0}$. The orange dotted and blue dashed lines denote the reference slope with $\propto B_{0}^{2}$ and the upper threshold $\epsilon_{\text {shear }}$. (b) $B_{0}$-dependence of $\mathcal{L}_{\mathrm{MRI}}$ (red circles and green cross). The blue dashed line denotes $\mathcal{L}_{\mathrm{MRI}}$ expected in the PNS with $3 \Omega_{0}$. The gray shades are constraint on $B_{0}$ and $L_{\mathrm{MRI}}$ for the extreme case.

the pulsar-sized magnetic field $\left(10^{12}-10^{13} \mathrm{G}\right)$ threads the post-collapse core in the rapidly rotating PNS.

Recently, the effects of the turbulence on the supernova dynamics are getting a lot more attention in numerical modeling of CCSNe. Not only the turbulent heating discussed here, the angular momentum transport and the material mixing in the meridional plane due to the turbulence should also play a crucial role in facilitating CCSNe (e.g., Murphy \& Meakin 2011; Hanke et al.|2012; Handy et al. 2014; Sawai \& Yamada 2014). Aided by the detailed analysis of the MRI in the linear phase (e.g., Guilet et al. 2014), future higher resolution 3D numer- ical modeling of CCSNe will uncover hidden properties of the MHD turbulence in the supernova core. We have attempted the very first step toward such models in this study.

We acknowledge the anonymous reviewer for constructive comments. Computations were carried on XC30 at NAOJ. This work was supported by the Grants-in-Aid for the Scientific Research from the Ministry of Education, Science and Culture of Japan (Nos. 24740125, 26870823, 24244036, 24103006, 26707013)

\section{REFERENCES}

Akiyama, S., Wheeler, J.C., Meier, D.L., \& Lichtenstadt, I. 2003, Astrophys. J., 584, 954

Balbus, S.A., \& Hawley, J.F. 1994, MNRAS, 266, 769

Balbus, S.A., \& Hawley, J.F. 1998, RvMP, 70, 1

Burrows, A., Dessart, L., Livne, E., Ott, C. D., \& Murphy, J. 2007, ApJ, 664, 416

Clarke, D. A. 1996, ApJ, 457, 291

Evans, C. R. \& Hawley, J. F. 1988, ApJ, 332, 659

Endeve, E., Cardall, C. Y., Budiardja, R. D., et al. 2012, ApJ, 751,26

Guilet, J., Mueller, E., \& Janka, H.-T. 2014, arXiv:1410.1874

Hanke, F., Marek, A., Müller, B., \& Janka, H.-T. 2012, ApJ, 755, 138

Handy, T., Plewa, T., \& Odrzywołek, A. 2014, ApJ, 783, 125

Kotake, K., Takiwaki, T., Suwa, Y., et al. 2012, Advances in Astronomy, vol. 2012, id. 428757

Masada, Y., Sano, T., \& Takabe, H. 2006, ApJ, 641, 447

Masada, Y., Sano, T., \& Shibata, K. 2007, ApJ, 655, 447

Masada, Y., Takiwaki, T., Kotake, K., \& Sano, T. 2012, ApJ, 759,110
Moffatt, H.K. 1978, Magnetic field generation in electrically conducting fluids, Cambridge University Press, 1978. 353 p.

Mösta, P., Richers, S., Ott, C.D., et al. 2014, ApJ, 785, L29

Murphy, J.W., \& Meakin, C. 2011, ApJ, 742, 74

Obergaulinger, M., Aloy, M.A., Müller, E. 2006, A\&A, 450, 1107

Obergaulinger, M., Cerdá-Durán, P., Müller, E., \& Aloy, M. A. 2009, A\&A, 498, 241

Ott, C. D., Burrows, A., Thompson, T. A., Livne, E., \& Walder, R. 2006, ApJS, 164, 130

Sawai, H., Yamada, S., \& Suzuki, H. 2013, ApJ, 770, L19

Sawai, H., \& Yamada, S. 2014, ApJ, 784, L10

Sano, T., Inutsuka, S., \& Miyama, S.M. 1998, ApJ, 506, L57

Shen, H., Toki, H., Oyamatsu, K., \& Sumiyoshi, K. 1998, Nuclear Physics A, 637, 435

Suzuki, T. K., Muto, T., \& Inutsuka, S.-i. 2010, ApJ, 718, 1289

Spruit, H.C. 2008, AIP Conference Proceedings, 983, 391

Takiwaki, T., Kotake, K., \& Sato, K. 2009, ApJ, 691, 1360

Takiwaki, T., \& Kotake, K. 2011, ApJ, 743, 30

Takiwaki, T., Kotake, K., \& Suwa, Y. 2014, ApJ, 786, 83

Thompson, T. A., Quataert, E., \& Burrows, A. 2005, ApJ, 620, 861

Winteler, C., Käppeli, R., Perego, A., et al. 2012, ApJ, 750, L22 